\title{
SOLID DISPERSION TO IMPROVE DISSOLUTION OF DRUG PRODUCT
}

\author{
Asma Huq \\ Department of Pharmacy, The University of Asia Pacific, Bangladesh
}

\section{Introduction}

The term 'solid dispersion' has been utilized to describe a family of dosage forms whereby the drug is dispersed in a biologically inert matrix, usually with a view to enhancing oral bioavailability. It may be defined as the dispersion of one or more active ingredients in an inert carrier matrix at solid-state prepared by the melting (fusion), solvent or melting-solvent method. In practice, these dosage forms have been traditionally regarded as being synonymous with systems whereby the in vitro release of the drug is enhanced compared to conventional dosage forms, with concomitant implications for in vivo release. Furthermore, the carrier used has, again traditionally, been a water-soluble or water-miscible polymer such as polyethylene glycol (PEG) or polyvinylpyrrolidone (PVP) or low molecular weight materials such as sugars. However, the proliferation of publications in the area since the first solid dispersions were described $^{1}$ has led to a broadening of these definitions to include water insoluble matrices such as Gelucires and Eudragits that may yield either slow or rapid release or absorption.

Correspondence to Author:

Asmahuq12@yahoo.com 
Table 1 Types of Solid Dispersion

\begin{tabular}{|c|c|c|c|c|}
\hline \multicolumn{2}{|c|}{ Solid dispersion type } & \multirow{2}{*}{$\begin{array}{c}\text { Matrix } \\
* \\
\mathrm{C}\end{array}$} & \multirow{2}{*}{$\begin{array}{c}\text { Drug } \\
* * \\
\mathrm{C}\end{array}$} & \multirow{2}{*}{$\begin{array}{l}\text { Remarks } \\
\text { The first type of solid dispersion } \\
\text { prepared }\end{array}$} \\
\hline I & Eutectics & & & \\
\hline II & $\begin{array}{l}\text { Amorphous } \\
\text { precipitations in } \\
\text { crystalline matrix }\end{array}$ & $\mathrm{C}$ & $\mathrm{A}$ & Rarely encountered \\
\hline \multirow[t]{5}{*}{ III } & Solid solutions & & & \\
\hline & $\begin{array}{l}\text { Continuous solid } \\
\text { solutions }\end{array}$ & $\mathrm{C}$ & $\mathrm{M}$ & $\begin{array}{l}\text { Miscible at all composition, never } \\
\text { prepared }\end{array}$ \\
\hline & $\begin{array}{l}\text { Discontinuous solid } \\
\text { solutions }\end{array}$ & $\mathrm{C}$ & $\mathrm{M}$ & $\begin{array}{l}\text { Partially miscible, } 2 \text { phases even } \\
\text { though drug is molecularly } \\
\text { dispersed. }\end{array}$ \\
\hline & $\begin{array}{l}\text { Substitutional solid } \\
\text { solutions }\end{array}$ & $\mathrm{C}$ & $\mathrm{M}$ & $\begin{array}{l}\text { Molecular diameter of drug (solute) } \\
\text { differs less than } 15 \% \text { from the matrix } \\
\text { (solvent) diameter. In that case the } \\
\text { drug and matrix are substitutional. } \\
\text { Can be continuous or discontinuous. } \\
\text { When discontinuous: } 2 \text { phases even } \\
\text { though drug is molecularly } \\
\text { dispersed. }\end{array}$ \\
\hline & $\begin{array}{l}\text { Interstitial solid } \\
\text { solutions }\end{array}$ & $\mathrm{C}$ & $\mathrm{M}$ & $\begin{array}{l}\text { Drug (solute) molecular diameter } \\
\text { less than } 59 \% \text { of matrix (solvent) } \\
\text { diameter. } \\
\text { Usually limited miscibility, } \\
\text { discontinuous. Example: Drug in } \\
\text { helical interstitial spaces of PEG. }\end{array}$ \\
\hline IV & Glass suspension & $\mathrm{A}$ & $\mathrm{C}$ & $\begin{array}{l}\text { Particle size of dispersed phase } \\
\text { dependent on cooling/evaporation } \\
\text { rate. Obtained after crystallization of } \\
\text { drug in amorphous matrix }\end{array}$ \\
\hline $\mathrm{V}$ & Glass suspension & $\mathrm{A}$ & $\mathrm{A}$ & $\begin{array}{l}\text { Particle size of dispersed phase } \\
\text { dependent on cooling/evaporation } \\
\text { rate many solid dispersions are of } \\
\text { this type }\end{array}$ \\
\hline VI & Glass solution & $\mathrm{A}$ & $\mathrm{M}$ & $\begin{array}{l}\text { Requires miscibility OR solid } \\
\text { solubility, complex formation or } \\
\text { upon fast cooling OR evaporation } \\
\text { during preparation, many (recent) } \\
\text { examples especially with PVP }\end{array}$ \\
\hline
\end{tabular}

${ }^{*} \mathrm{~A}$ : matrix in the amorphous state, C: matrix in the crystalline state

**: A: drug dispersed as amorphous clusters in the matrix, C: drug dispersed as crystalline particles in the matrix, M: drug molecularly dispersed throughout the matrix 


\section{Proposed structures of solid dispersions}

The dispersions have traditionally been formed by heating mixes of the drug and carrier to the molten state (although whether this molten mix is a suspension or solution is usually not defined) followed by resolidification via cooling. Alternative methods involve dissolving the components in a mutual volatile solvent followed by evaporation or dissolving the drug in a solvent such as propylene glycol and adding that to the molten carrier. Other approaches include melt-extrusion methods that appear to offer a number of interesting opportunities. Irrespective of the methodology used, the question as to the physical nature of the dispersion remains unanswered in many cases. Classically, Chiou and Riegelman ${ }^{2}$ defined a number of possibilities. These include eutectic systems, whereby on cooling the molten mix the system forms a microfine dispersion of the two components with a concomitant decrease in melting point. This has been a favoured explanation for several systems, particularly in the light of DSC studies that have frequently been reported to show a eutectic melting point and a lowering of the melting points of the principle components. However, some caution is required in this interpretation for a number of reasons. In the first instance, it is essential to bear in mind that unless one is exactly at the eutectic composition, the system will contain a mixture of the microfine dispersion and one or other component as a separate phase, as indicated in Fig. 1a. Indeed, as one cools from the melt of any composition other than that corresponding to the eutectic, one component will progressively solidify, thereby rendering the remaining liquor richer in the other component until the eutectic composition is reached, at which point the remaining liquid will solidify as a fine dispersion. Consequently, if the reported systems are indeed eutectics it is necessary to appreciate the complex nature of the mixes used in practice. The second issue is that the polyethylene glycols used for the majority of solid dispersion studies (molecular weight 4000-20,000) may exist in more than one crystal form, exhibiting multiple melting points in the region of 55-65 $\mathrm{C}$. It has be en suggested that many of the dual melting points described in the literature ascribed to eutectic behaviour may in fact be chain folded forms of the PEG itself. Thirdly, it is arguably essential to compare the melting behaviour of the solid dispersion to that of a physical mix of the drug and carrier, as many studies have indicated that the phase diagrams of the two systems may be extremely similar. Indeed, the presence of the carrier in the molten state may itself lower the melting point of the drug. Consequently, the detection of melting point lowering and, in the case of PEGs, the appearance of a lower temperature melting peak, does not necessarily indicate the presence of a eutectic. While some systems must inevitably form eutectics, the number of studies that have demonstrated unequivocally that a eutectic is present is in fact very limited. The second common explanation is that of a solid solution, whereby the drug is present as a molecular dispersion within the carrier. This is a fully feasible explanation but again caution is required in terms of the detection of such systems. In practice the majority of such systems are likely to show only partial miscibility, hence the drug may only be in 'solution' at low concentrations (Fig. 1b), although it is appreciated that partial miscibility could in theory involve quite extensive drug incorporation at a molecular level. Nevertheless unequivocal demonstration of solid solubility is not as simple as one may imagine. For the reasons outlined above the melting point of a drug may be lowered and broadened so as to make it undetectable using DSC. Similarly, XRD analysis needs to be conducted in comparison to physical mixes of identical composition to ascertain whether the lack of appearance of drug peaks is due to solid solution formation or is simply a function of the sensitivity of the instrument. Thirdly, the drug may be present as a dispersion in a glassy matrix. This is certainly the case with amorphous polymers such as PVP and is probably also of relevance in many cases to semicrystalline materials such as PEGs. Again, questions still remain as to whether the drug is dispersed on a molecular basis, is present as a separate amorphous phase or is present as a separate crystalline phase (or some combination of these). In some respects this is perhaps the area in which the most progress has been made, as the 
work by the group of and others over the last decade has provided a more thorough basis by which to study and understand amorphous systems. This is arguably ironic as such systems may be expected to be more complex than crystalline dispersions. Finally, complex formation has been suggested as a further possibility. Clearly this is applicable to materials such as cyclodextrins and may also be of relevance to PVP and other carriers. A further category that has been suggested since the review of Chiou and Riegelman ${ }^{2}$ has been that of monotectic systems. These systems were suggested on the basis of the frequent observation that many reported eutectics had eutectic points that appeared to be convergent with the melting point of the pure material, as indicated in Fig. 1c. Such systems have been reported in the alloy literature but later studies have indicated that, rather than indicating specific equilibrium phase behaviour, such diagrams may be simply a reflection of a completely non-interacting system, whereby the drug is simply present as a separate phase within the carrier. This was exemplified by a study whereby the model drug (paracetamol) was found to simply crystallise as a separate phase on cooling, as demonstrated by hot stage microscopy, the lowering of drug melting point being a simple reflection of the presence of molten carrier. Overall, therefore, there still remain numerous questions regarding the physical nature of solid dispersions, despite the chemical simplicity of these systems. There is an argument that, at least in the case of PEGs and other largely crystalline carriers, the system may be simpler than has been assumed, with the two components simply existing as separate phases. Similarly it could also be argued that hot stage microscopy observations during cooling is one of the most reliable methods for ascertaining the solid state structure, especially given the propensity of DSC to demonstrate effects that are a function of the temperature programme rather than being a direct reflection of the solid state structure at room temperature. 
Volume 2, Issue 1: January 2013
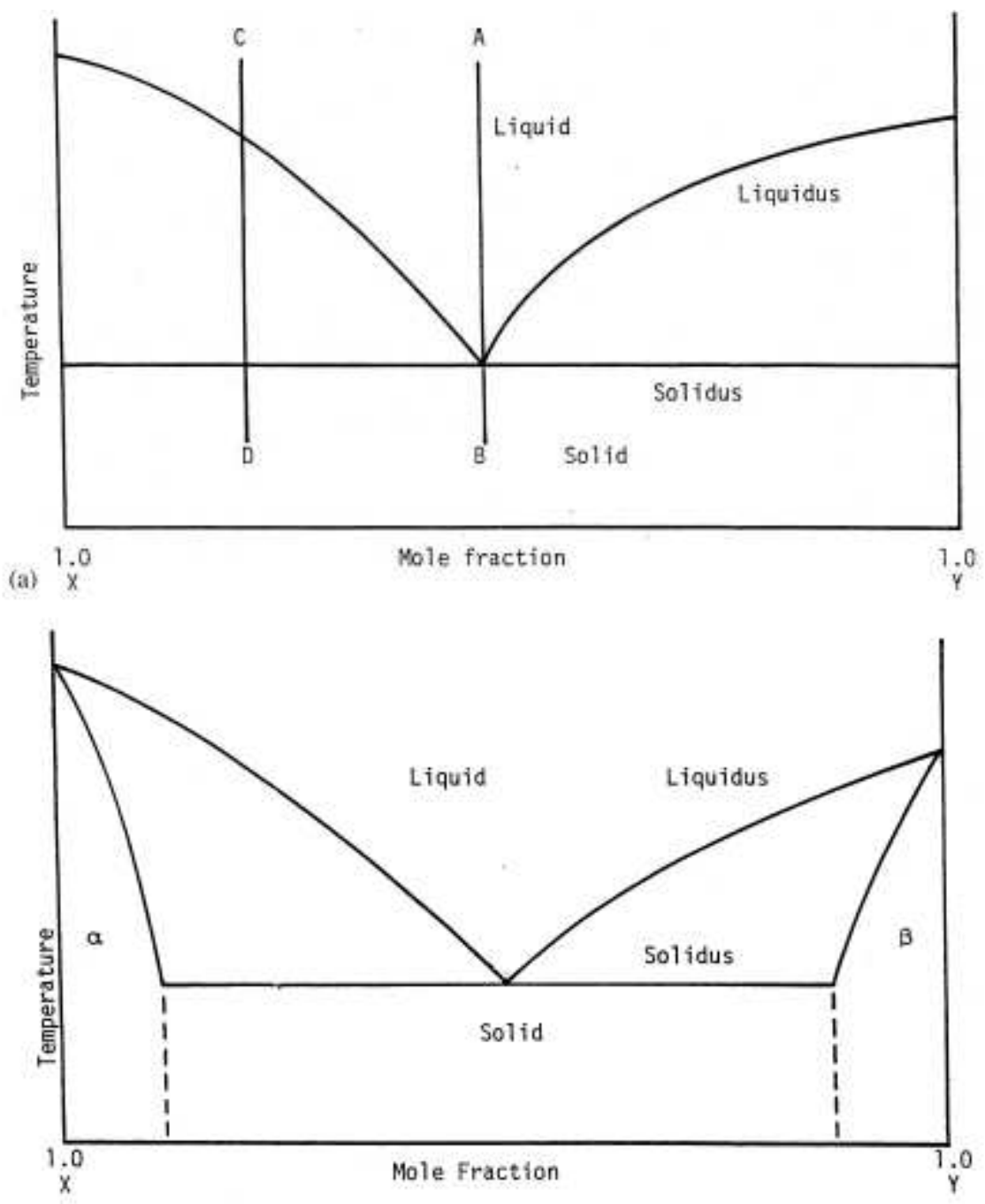

(b) $\alpha$ - Solid solution of $Y$ in $X$

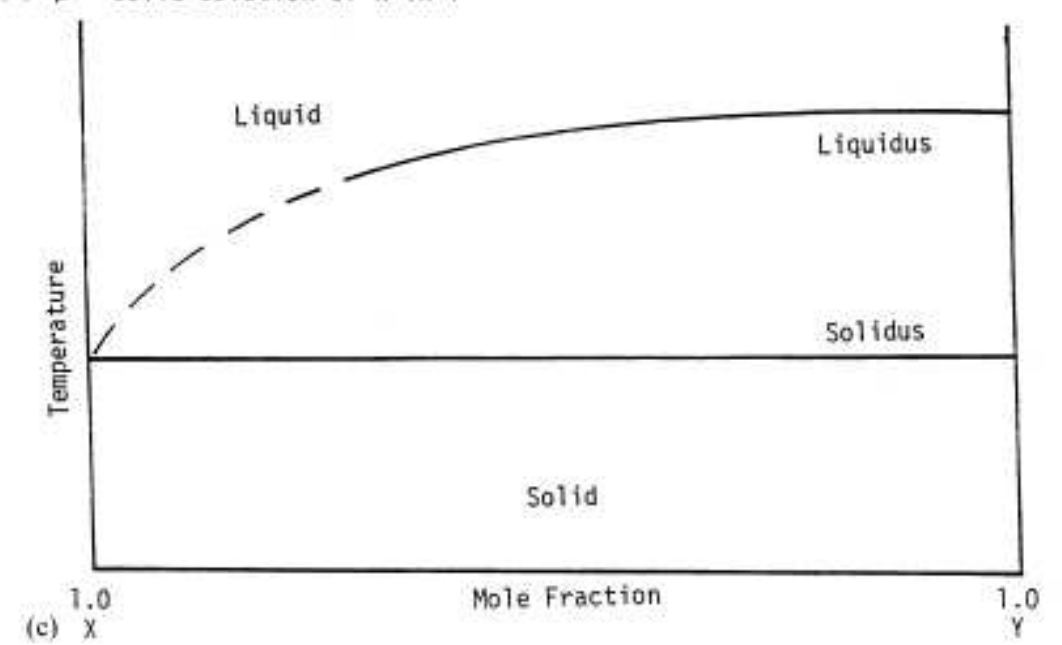

Figure 1 (a) Schematic eutectic phase diagram, showing effect of cooling at the eutectic point (AtoB) where by only the eutectic solidifies. This is compared to cooling at an alternative 
composition (CtoD) where by component $X$ solidifies during cooling, leaving the remaining liquor richer in $\mathrm{Y}$ until the eutectic temperature/composition is reached. (b) Schematic solid solution phase diagram (partial miscibility), showing regions of solid solubility at the extremes of composition. (c) Schematic monotectic phase diagram, showing convergence of the liquidus with the melting point of one component.

\section{Drug release from solid dispersions}

\section{Particle size reduction and reduced agglomeration}

These may be usefully considered together as both are related to increases in the exposed surface area of the drug. Size reduction has been classically considered to be a result of eutectic or solid solution formation; it is worth noting that this mechanism suggests an intrinsic link between solid state structure and release. Similarly it has been suggested that the presentation of particles to the dissolution medium as physically separate entities may reduce aggregation. In addition, many of the carriers used for solid dispersions may have some wetting properties, hence it is reasonable to suggest that improved wetting may lead to reduced agglomeration and hence increased surface area.

\section{Increased solubility or dissolution rate of the drug}

Many of the carriers used may increase the solubility of the drug. There has been some debate over this mechanism as solubility studies have indicated that at the concentrations used for in vitro experiments the carriers often elicit minimal solubility increases. This does, however, work on the assumption that the concentration of the carrier after complete dissolution in the water bath (e.g. $0.5 \mathrm{~g} / \mathrm{l})$ may be used as a model of the behaviour at the dissolving surface. Similarly, the carrier and drug may form a soluble complex, as is well established for cyclodextrins, although the evidence for this occurring with other carriers is weaker. Finally, changes to the physical properties of the drug such as degree of crystallinity and polymorphic form may also be considered under this category.

There have been two apparently conflicting lines of research along these lines. In the first instance, Corrigan ${ }^{1}$ provided a very valuable contribution by not only measuring the dissolution rate of the incorporated drug but also assessing that of the polymer itself, in this case PEG. The author found that the dissolution rate of the drug in the polymer and the polymer alone were in fact equivalent, leading to the suggestion of carrier-controlled dissolution whereby the dissolution rate of the drug is controlled by that of the inert carrier. This finding was supported by the work of Dubois and Ford ${ }^{1}$ who noted that the dissolution rates of a range of drugs in a single carrier, prepared under comparable conditions, were identical in most cases. This again implies that it is the dissolution rate of the carrier and not the drug that may dominate the process. Similarly, a study by Craig and Newton ${ }^{1}$ indicated that a log-linear relationship existed between the (measured as opposed to nominal) molecular weight of the PEG carrier and the dissolution rate, again implying that the properties of the polymer were dominating the dissolution process. Corrigan has suggested that carrier-controlled dissolution may be modelled in terms of the approach outlined by Higuchi, whereby the dissolution of two-component systems is considered. Upon exposure to the solvent both components dissolve at rates proportional to their solubilities (Cs) and diffusion coefficients $(D)$ in the dissolving medium, as predicted for single component systems by the well-known Noyes-Whitney equation. However this model predicts that the interfacial layer between the dissolving front and the solvent will become depleted in the more rapidly dissolving component, leading to the creation of a surface 
layer rich in one component through which the other must diffuse prior to release into the bulk phase (Fig. 2).

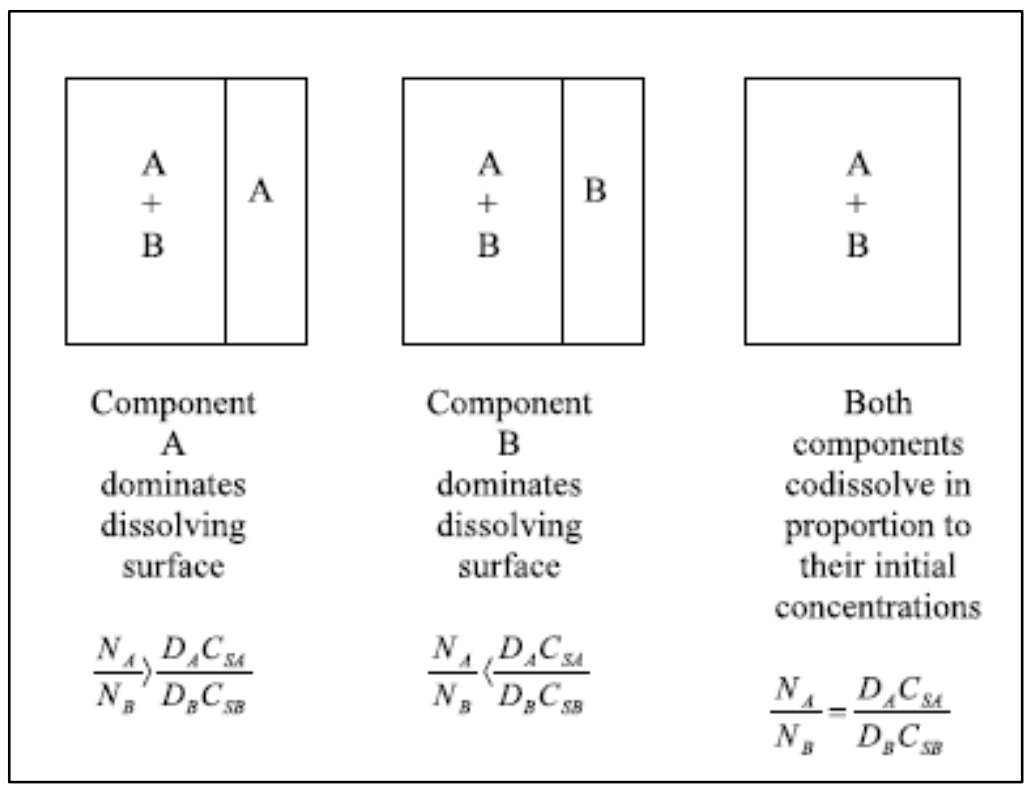

Figure 2-Schematic representation of the dissolution model for a two component system

More specifically the model predicts that one component (for example A) will form such a surface layer when

$$
\frac{N_{\mathrm{A}}}{N_{\mathrm{B}}}>\frac{D_{\mathrm{A}} C_{\mathrm{SA}}}{D_{\mathrm{B}} C_{\mathrm{SB}}}
$$

where $N$ is the proportion of each component and the subscripts $A$ and $B$ refer to the two components, respectively. Under these circumstances the dissolution rates will be given by

$$
G_{\mathrm{A}}=\frac{D_{\mathrm{A}} C_{\mathrm{SA}}}{h}
$$

and

$$
G_{\mathrm{B}}=\frac{N_{\mathrm{B}}}{N_{\mathrm{A}}} G_{\mathrm{A}} \ldots \ldots \ldots . . . \mathrm{Eq}-6
$$

where $G$ is the dissolution rate/unit area and $h$ is the diffusion layer thickness. In other words the model predicts that the dissolution rate of the minor component will be determined by that of the component in excess (although it should be noted that 'minor' is more precisely defined in terms of Eq. (4) rather than simply the weight fraction present). This may in turn be applied to solid disperse systems by arguing that when the drug is present as a 'minor' component (which is almost invariably the case) the dissolution of that drug will be dominated by the dissolution 
behaviour of the carrier, as is indeed seen in practice. Interestingly, the predicted component ratio at which dominance changes between the two components is given by

$$
\frac{N_{\mathrm{A}}}{N_{\mathrm{B}}}=\frac{D_{\mathrm{A}} C_{\mathrm{SA}}}{D_{\mathrm{B}} C_{\mathrm{SB}}} \ldots \ldots \ldots \text { Eq } 7
$$

This therefore predicts that if a drug $(B)$ has a very low solubility in relation to that of the polymer (A) then the drug loading up to which carrier-controlled dissolution will apply will be similarly low, while a more soluble drug will show carrier-controlled dissolution up to a higher drug loading (assuming similar diffusion coefficients). This would appear to contradict the observations of Dubois and Ford ${ }^{1}$, who noted that phenacetin $(0.77 \mathrm{mg} / \mathrm{ml}$ solubility) showed a more limited range of carrier-controlled dissolution (upto $5 \%$ loading) than did the less soluble $(0.04 \mathrm{mg} / \mathrm{ml}$ ) indomethacin (up to $10 \%$ drug loading). This could be due to diffusion coefficient effects or else to differences in the dispersion profiles of the two drugs within the polymer.

Leading on from these studies, Lloyd et al. argued that if dissolution was dominated by the properties of the carrier and not the drug (at least in some cases) then the physical form of the drug should be irrelevant to the release rate ${ }^{1}$. These authors examined the release of paracetamol from PEG 6000 dispersions, using different drug size fractions in the initial preparation process and different manufacturing methods which were known to alter the physical properties of the drug. First inspection of the dissolution data indicated a higher release from the larger size fraction systems. However, these authors also measured the concentration of drug at the dissolving surface, finding that settling had occurred during the solidification process on cooling from the melt. Once this had been corrected for (see Eq. 6), the dissolution rates were found to be independent of manufacturing conditions or initial particle size. This therefore confirmed that for these systems the physical form of the drug was unimportant as far as the release rate was concerned but also highlighted the danger of settling leading to higher effective concentrations of drug being present at the dissolving surface than may be anticipated from the total drug content.

However, while these studies, described together, give the impression of there being a common unifying mechanism underpinning release, there have been a number of papers suggesting that other mechanisms may be of relevance. For example, Sjo" kvist and Nystro"m measured the particle size of the griseofulvin particles released from the dispersions and produced strong evidence that dissolution rate enhancement was a direct function of the size of the released particles ${ }^{1}$. In an attempt to reconcile these contradictions Sjo" kvist-Saers and Craig used a homologous series of drugs (para-aminobenzoates) in PEG 6000 in an attempt to interrelate the solid state structure, drug solubility and dissolution rate. These authors noted that there was a linear relationship between the intrinsic dissolution rate of the model drugs in the dispersions and the drug solubility, clearly linking the properties of the drug (and not the polymer) to the dissolution rate; it may be helpful at this stage to refer to such behaviour as drug-controlled dissolution as opposed to carrier-controlled dissolution. It was also noted that as the concentration of the drug increased the dissolution rate became effectively independent of composition and very similar to the drug alone (Fig. 2); in this respect therefore the behaviour corresponds to the Higuchi model when the drug is the dominant component. However the interrelationship between the dissolution rate and the solubilities of the drugs at high polymer contents runs contrary to what one would expect from the Higuchi model; if the dissolution was carrier controlled the drug properties should make no difference to the dissolution rate. 
Overall, therefore, there appear to be two sets of observations with regard to the mechanism of drug release from solid dispersions. In the first instance, some systems appear to show carrier controlled release whereby, at least at low drug loadings, the rate of release is controlled by that of the carrier and is independent of drug properties. Secondly some systems show release behaviour that is dependent on the properties of the drug rather the polymer, even at low drug loadings.

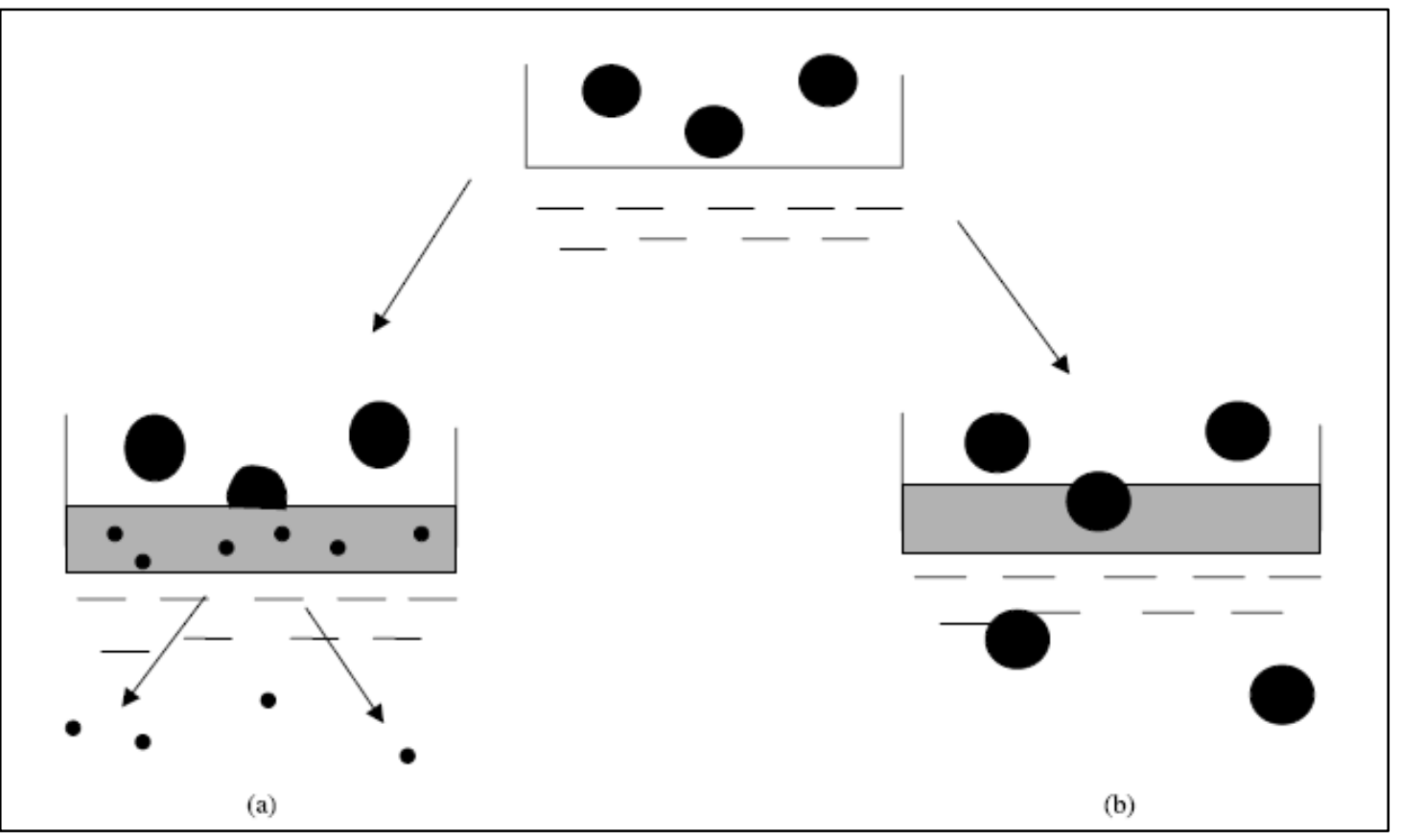

Figure 3-Schematic diagram showing the fate of drug particles during the dissolution process. (a) Carrier-controlled dissolution, whereby the drug dissolves into the concentrated carrier layer prior to release and (b) drug-controlled dissolution whereby the drug is released effectively intact into the dissolution medium. Large spheres represent undissolved drug particles, small spheres partially dissolved drug particles, shaded regions correspond to hydrated material.

\section{Possible mechanism of dissolution from solid dispersions}

Dissolution mechanism may be described by two ways- carrier controlled dissolution and drug controlled dissolution. In Fig. 3a, the process associated with carriercontrolled dissolution is described. In this instance the particles dissolve into the polymer-rich diffusion layer at a sufficiently rapid rate that there is insufficient time for the particles to be released intact into the medium. Consequently, the drug is

molecularly dispersed within this concentrated layer. However, the viscosity of the layer is such that drug diffusion is very slow as predicted by the well-known Stokes-Einstein equation

$$
D=\frac{k T}{6 \pi \eta r}
$$


where $k$ is Boltzmann's constant, _ is the viscosity and $r$ is the radius of the diffusing molecule. Consequently the rate-limiting step to dissolution of the drug becomes the release of the polymeritself and hence Eq- 6 becomes applicable to describe the release of the drug. It should be noted that the dissolution of a water soluble polymer may not be modelled by simple diffusion equations with complete confidence as such polymers do not show saturation solubility as such but rather will swell and sorb water to produce a continuum of concentrations between the solid surface and the bulk medium.

The second scenario, that of drug-controlled dissolution, is outlined in Fig. 3b. In this case dissolution into the polymer diffusion layer is comparatively slow and the drug is released as solid particles. Consequently the dissolution will not be associated with the polymer but will instead be dominated by the properties (size, physical form, etc.) of the drug itself. This may still lead to considerable improvements in dissolution compared to conventional dosage forms due to the higher surface area associated the particles and the possibility of improved wetting and decreased agglomeration.

The question therefore arises as to why some drugs will follow the scenario shown in Fig. $3 a$ and others $3 \mathrm{~b}$. The most likely explanation must be the tendency of the drug to dissolve into the concentrated polymer diffusion layer. As stated previously, the solubility of drugs in low concentrations of carrier solutions does not tend to be significantly enhanced compared to that in water alone. However, several studies have demonstrated that the drug solubility increases disproportionately in higher concentration solutions. More specifically, a log-linear relationship has been described for several systems as predicted by the expression given for cosolvent systems

$$
\log S=\log S_{\mathrm{W}}+\sigma f
$$

where $S$ is the solubility of the solvent under investigations, $S_{w}$ is the solubility in water, $\sigma$ is a constant and $f$ is the proportion of cosolvent present in the system. It is therefore feasible that for many drug-carrier combinations the drug solubility in the concentrated layer is sufficiently high so as to allow dissolution to occur prior to the dissolving front of the composite solid reaching the particles. Once in solution in the diffusion layer, the viscosity is sufficiently high so as to render diffusion through the concentrated layer slow and the rate-limiting step to release becomes the diffusion of the carrier molecules into the bulk phase, as predicted by the Higuchi model. In the case of drugs whereby the solubilisation is low, however, the particles may be simply released partially or completely intact from the matrix, where upon dissolution occurs from the free particle surface.

Overall, therefore, the release mechanism will depend on whether the drug dissolves in the polymer diffusion layer rapidly or not which will in turn be dependent largely on the solubility of the drug in this layer. However, other considerations must also be borne in mind. For example, the hydrodynamics of the dissolution process may also play a role in determining the mechanism in that more rapid stirring speeds may favour drugcontrolled dissolution by enhancing the rate of polymer dissolution into the bulk in relation to drug dissolution into the diffusion layer. Similarlyby changing the physical form of the drug (e.g.size reduction), one could conceivably change the mechanism by altering the dissolution kinetics into the diffusion layer. 


\section{Manufacturing processes of Solid Dispersion}

Melting and solvent evaporation methods are the two major processes of preparing solid dispersions $^{3}$ (Figure 4).

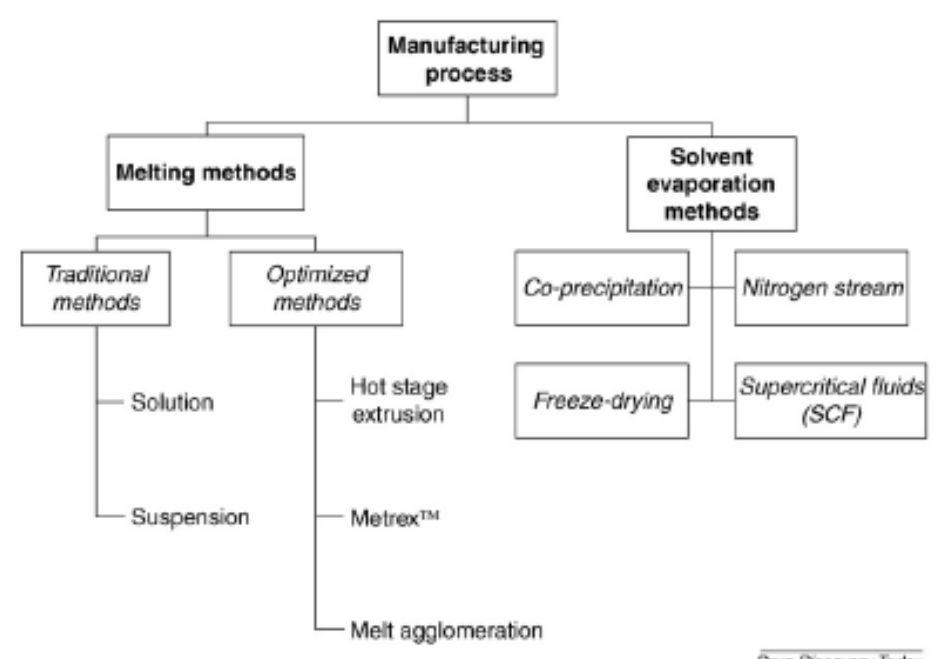

Figure 4- Manufacturing processes used to produce solid dispersions.

\section{Melting method}

Sekiguchi et al. ${ }^{4}$ were the first to use a melting method consisting of melting the drug within the carrier followed by cooling and pulverization of the obtained product. In the melting process, the molecular mobility of carrier is high enough to change the drug's incorporation. A common adaptation to the melting phase consists of suspending the active drug in a previously melted carrier, instead of using both drug and carrier in the melted state, reducing, therefore, the process temperature.

To cool and solidify the melted mixture, several processes such as ice bath agitation, stainless steel thin layer spreading followed by a cold draught, solidification on petri dishes at room temperature inside a dessicator, spreading on plates placed over dry ice, immersion in liquid nitrogen or stored in a dessicator were used. After cooling, the mixture must be pulverized regarding its handling ${ }^{5}$.

However, the use of high temperatures, and the fact that several drugs can be degraded by the melting process, can be a limitation of this method. The incomplete miscibility between drug and carrier that may occur, because of the high viscosity of a polymeric carrier in the molten state, is another limitation of this process. To avoid the melting method limitations, several modifications, like hot-stage extrusion, MeltrexTM or melt agglomeration were introduced to the original method.

Hot-stage extrusion consists of the extrusion, at high rotational speed, of the drug and carrier, previously mixed, at melting temperature for a small period of time. The resulting product isthen collected after cooling at room temperature and milled. A reduction in processing temperature can be achieved by the association of hot-stage extrusion with the use of carbon dioxide as a 
plasticizer, which broadens the application of hot-stage extrusion to thermally labile compounds. Solid dispersions of para-amino salicylic acid/ethylcellulose, itraconazole/PVP and itraconazole/ethylcellulose were successfully prepared by this technique. Moreover, it was observed that solid dispersions of itraconazole/inutec SP1 prepared by hot-stage extrusion presented itraconazole in a fully glassy state, whereas it was only partially glassy in solid dispersions prepared by spray drying ${ }^{6}$.

MeltrexTM is a patented solid dispersion manufacturing process, also on the basis of the melting process. The crucial elements in the MeltrexTM technology is the use of a special twin screw extruder and the presence of two independent hoppers in which the temperature can vary over a broad temperature range. This process permits a reduced residence time of the drug in the extruder, allowing a continuous mass flow and avoiding thermal stress to the drug and excipients. Additionally, it is possible that the application of this technique to protect drugs susceptible to oxidation and hydrolysis by complete elimination of oxygen and moisture from the mixture.

Melt agglomeration allows the preparation of solid dispersions in conventional high shear mixers. It is made by adding the molten carrier containing the drug to the heated excipients, by adding the molten carrier to a heated mixture of drug and excipients, or by heating a mixture of the drug, carrier and excipients to a temperature within or above the melting range of the carrier. It is also possible to produce stable solid dispersions by melt agglomeration in a rotary processor.

\section{Solvent evaporation method}

The solvent evaporation method consists of the solubilization of the drug and carrier in a volatile solvent that is later evaporated. In this method, the thermal decomposition of drugs or carriers can be prevented, since organic solvent evaporation occurs at low temperature ${ }^{7}$.

A basic process of preparing solid dispersions of this type consists of dissolving the drug and the polymeric carrier in a common solvent, such as ethanol, chloroform, or a mixture of ethanol and dichloromethane. Normally, the resulting films are pulverized and milled. The use of the carriers partially suspended, instead of dissolved, was also reported in the preparation of a solid dispersion of indometacin, in which the drug and ethylcellulose were dissolved in ethanol and HPMC was suspended ${ }^{7}$.

Differences in solvent evaporation processes are related to the solvent evaporation procedure, which usually include vacuum drying, heating of the mixture on a hot plate, slow evaporation of the solvent at low temperature, the use of a rotary evaporator, a stream of nitrogen, spraydrying, freeze-drying and the use of supercritical fluids (SCF)

Spray-drying is one of the most commonly used solvent evaporation procedures in the production of solid dispersions. It consists of dissolving or suspending the drug and carrier, then spraying it into a stream of heated air flow to remove the solvent . Van Drooge et al. ${ }^{8}$ prepared an alternative solid dispersion by spraying a povidone and diazepam solution into liquid nitrogen, forming a suspension that was then lyophilized.

The basic freeze-drying process consists of dissolving the drug and carrier in a common solvent, which is immersed in liquidnitrogen until it is fully frozen. Then, the frozen solution is further lyophilized. 
The use of SCF, substances existing as a single fluid phase above their critical temperature and critical pressure, was shown to be efficient in obtaining solid dispersions. It ensured a very fine dispersion of the hydrophobic drug in the hydrophilic carrier. Carbon dioxide $\left(\mathrm{CO}_{2}\right)$ is the most commonly used SCF because is chemically inert, non-toxic and nonflammable. This technique consists of dissolving the drug and the carrier in a common solvent that is introduced into a particle formation vessel through a nozzle, simultaneously with $\mathrm{CO}_{2}$. When the solution is sprayed, the solvent is rapidly extracted by the SCF, resulting in the precipitation of solid dispersion particles on the walls and bottom of the vessel. The use of processes using SCF reduces particle size, residual solvent content, without any degradation, and often results in high yield ${ }^{7}$.

Another common process is the co-precipitation method, in which a non-solvent is added dropwise to the drug and carrier solution, under constant stirring. In the course of the nonsolvent addition, the drug and carrier are co-precipitated to form microparticles. At the end, the resulted microparticle suspension is filtered and dried.

Spin-coated films is a new process to prepare solid dispersions by the solvent evaporation method, which consists of dissolving drug and carrier in a common solvent that is dropped onto a clean substrate highly spinned. Solvent is evaporated during spinning. This process is indicated to moisture sensitive drugs since it is performed under dry conditions. The use of organic solvents, the high preparation cost and the difficulties in completely removing the solvent are some of the disadvantages associated with solvent evaporation methods. Moreover, it is also possible that slight alterations in the conditions used for solvent evaporation may lead to large changes in product performance.

\section{Advantages of solid dispersions over other strategies to improve bioavailability of poorly water soluble drugs}

Improving drug bioavailability by changing their water solubility has been possible by chemical or formulation approaches.

Chemical approaches to improving bioavailability without changing the active target can be achieved by salt formation or by incorporating polar or ionizable groups in the main drug structure, resulting in the formation of a pro-drug. Solid dispersions appear to be a better approach to improve drug solubility than these techniques, because they are easier to produce and more applicable. For instance, salt formation can only be used for weakly acidic or basic drugs and not for neutral. Furthermore, it is common that salt formation does not achieve better bioavailability because of its in vivo conversion into acidic or basic forms. Moreover, these type of approaches have the major disadvantage that the sponsoring company is obliged to perform clinical trials on these forms, since the product represents a NCE.

Formulation approaches include solubilization and particle size reduction techniques, and solid dispersions, among others. Solid dispersions are more acceptable to patients than solubilization products, since they give rise to solid oral dosage forms instead of liquid as solubilization products usually do. Milling or micronization for particle size reduction are commonly performed as approaches to improve solubility, on the basis of the increase in surface area. Solid dispersions are more efficient than these particle size reduction techniques, since the latter have a particle size reduction limit around $2-5 \mathrm{~mm}$ which frequently is not enough to improve considerably the drug solubility or drug release in the small intestine and, consequently, to improve the bioavailability ${ }^{9}$. Moreover, solid powders with such a low particle size have poor 
mechanical properties, such as low flow and high adhesion, and are extremely difficult to handle.

\section{The advantageous properties of solid dispersions}

\section{Particles with reduced particle size}

Molecular dispersions, as solid dispersions, represent the last state on particle size reduction, and after carrier dissolution the drug is molecularly dispersed in the dissolution medium. Solid dispersions apply this principle to drug release by creating a mixture of a poorly water soluble drug and highly soluble carriers. A high surface area is formed, resulting in an increased dissolution rate and, consequently, improved bioavailability ${ }^{7}$.

\section{Particles with improved wettability}

A strong contribution to the enhancement of drug solubility is related to the drug wettability improvement verified in solid dispersions. It was observed that even carriers without any surface activity, such as urea improved drug wettability. Carriers with surface activity, such as cholic acid and bile salts, when used, can significantly increase the wettability properties of drugs. Moreover, carriers can influence the drug dissolution profile by direct dissolution or co-solvent effects $^{10}$. Recently, the inclusion of surfactants in the third generation solid dispersions reinforced the importance of this property.

\section{Particles with higher porosity}

Particles in solid dispersions have been found to have a higher degree of porosity. The increase in porosity also depends on the carrier properties, for instance, solid dispersions containing linear polymers produce larger and more porous particles than those containing reticular polymers and, therefore, result in a higher dissolution rate. The increased porosity of solid dispersion particles also hastens the drug release profile ${ }^{11}$.

\section{Drugs in amorphous state}

Poorly water soluble crystalline drugs, when in the amorphous state tend to have higher solubility. The enhancement ofdrug release can usually be achieved using the drug in its amorphous state, because no energy is required to break up the crystal lattice during the dissolution process. In solid dispersions, drugs are presented as supersaturated solutions after system dissolution, and it is speculated that, if drugs precipitate, it is as a metastable polymorphic form with higher solubility than the most stable crystal form ${ }^{7}$.

For drugs with low crystal energy (low melting temperature or heat of fusion), the amorphous composition is primarily dictated by the difference in melting temperature between drug and carrier. For drugs with high crystal energy, higher amorphous compositions can be obtained by choosing carriers, which exhibit specific interactions with them. 


\section{Strategies to avoid drug recrystallization}

Recrystallization is the major disadvantage of solid dispersions. As amorphous systems, they are thermodynamically unstable and have the tendency to change to a more stable state under recrystallization.

Molecular mobility is a key factor governing the stability of amorphous phases, because even at very high viscosity, below the glass transition temperature $(\mathrm{Tg})$, there is enough mobility for an amorphous system to crystallize over pharmaceutically relevant time scales. Furthermore, it was postulated that crystallization above $\mathrm{Tg}$ would be governed by the configurational entropy, because this was a measure of the probability of molecules being in the appropriate conformation, and by the mobility, because this was related to the number of collisions per unit time ${ }^{7}$.

Several experiments have been conducted to understand the stabilization of solid dispersions. Recent studies observed very small reorientation motions in solid dispersions showing a detailed heterogeneity of solid dispersionsanddetecting the sub-glass transition beta-relaxation as well as alpha-relaxation, which may lead to nucleation and crystal growth. Molecular mobility of the amorphous system depends, not only on its composition, but also on the manufacturing process as stated by Bhugra et al. ${ }^{12}$. Solid dispersions exhibiting high conformational entropy and lower molecular mobility are more physically stable.

Polymers improve the physical stability of amorphous drugs in solid dispersions by increasing the $\mathrm{Tg}$ of the miscible mixture, thereby reducing the molecular mobility at regular storage temperatures, or by interacting specifically with functional groups of the drugs. For a polymer to be effective in preventing crystallization, it has to be molecularly miscible with the drug. For complete miscibility, interactions between the two components are required. It is recognized that the majority of drugs contain hydrogen-bonding sites, consequently, several studies have shown the formation of ion-dipole interactions and intermolecular hydrogen bonding between drugs and polymers, and the disruption of the hydrogen bonding pattern characteristic to the drug crystalline structure. These lead to a higher miscibility and physical stability of the solid dispersions. Specific drug polymer interactions were observed by Teberekidis et al. ${ }^{7}$, showing that interaction energies, electron density, and vibrational data revealed a stronger hydrogen bond of felodipine with PVP than with PEG, which was in agreement with the dissolution rates of the corresponding solid dispersions.

Other studies have shown stabilization in systems where hydrogen-bonding interactions are not possible, because of the chemistry of the system. Vippagunta et al. ${ }^{7}$ concluded that fenofibrate does not exhibit specific interactions with PEG, independent of the number of hydrogen bonds donating groups presented. The same conclusion was achieved by Weuts et al. $^{13}$ in the preparation of solid dispersions of loperamide with PVP K30 and PVP VA64, in which, hydrogen bonds were no absolute condition to avoid crystallization.

Konno et al. ${ }^{14}$ determined the ability of three different polymers, PVP, HPMC and hydroxypropylmethylcellulose acetate succinate to stabilize amorphous felodipine, against crystallization. The three polymers inhibited crystallization of amorphous felodipine by reducing the nucleation rate ${ }^{14}$. It was speculated that these polymers affect nucleation kinetics by increasing their kinetic barrier to nucleation, proportional to the polymer concentration and independent of the polymer physiochemical properties ${ }^{14}$. 
The strategies to stabilize the solid dispersions against recrystallization strongly depend on the drug properties and a combination of different approaches appears to be the best strategy to overcome this drawback. Third generation solid dispersions intend to connect several strategies to overcome the drug recrystallization, which has been the major barrier to the solid dispersions marketing success.

\section{Disadvantages of Solid Dispersion}

Despite extensive expertise with solid dispersions, they are not broadly used in commercial products, mainly because there is the possibility that during processing (mechanical stress) or storage(temperature and humidity stress) the amorphous state may undergo crystallization ${ }^{7}$. The effect of moisture on the storage stability of amorphous pharmaceuticals is also a significant concern, because it may increase drug mobility and promote drug crystallization. Moreover, most of the polymers used in solid dispersions can absorb moisture, which may result in phase separation, crystal growth or conversion from the amorphous to the crystalline state or from a metastable crystalline form to a more stable structure during storage. This may result in decreased solubility and dissolution rate. Therefore, exploitation of the full potential of amorphous solids requires their stabilization in solid state, as well as during in vivo performance.

Another drawback of solid dispersions is their poor scale-up for the purposes of manufacturing.

\section{Conclusion}

Solid dispersion is discussed in this article in details including method, mechanism. Now solid dispersion can be a solution of obstacle of solubility. Though there are difficulties in scale up using solid dispersion, that difficulties can be solved by studying case by case.

\section{References}

1. Duncan Q.M. Craig, 2001. The mechanisms of drug release from solid dispersions in water-oluble polymers. Int. J. Pharm., 231, 131-144

2. Chiou, W. L., Riegelman, S., 1971. Pharmaceutical application of solid dispersion systems. Journal of pharmaceutical sciences 60 (9) 1281

3. Vilhelmsen, T. et al. (2005) Effect of a melt agglomeration process on agglomerates containing solid dispersions. Int. J. Pharm. 303, 132-142

4. Sekiguchi, K. and Obi, N. (1961) Studies on absorption of eutectic mixtures. I. A comparison of the behavior of eutectic mixtures of sulphathiazole and that of ordinary sulphathiazole in man. Chem. Pharm. Bull. 9, 866-872 
5. Owusu-Ababio, G. et al. (1998) Comparative dissolution studies for mefenamic acidpolyethylene glycol solid dispersion systems and tablets. Pharm. Dev. Technol. 3, 405-412

6. Van den Mooter, G. et al. (2006) Evaluation of Inutec SP1 as a new carrier in the formulation of solid dispersions for poorly soluble drugs. Int. J. Pharm. 316, 1-6

7. Vasconcelos, T. et al. (2007) Solid dispersions as strategy to improve oral bioavailability of poor water soluble drugs. Drug Discovery Today. Volume-12 (1068-1075)

8. van Drooge, D.J. et al. (2006) Characterization of the molecular distribution of drugs in glassy solid dispersions at the nano-meter scale, using differential scanning calorimetry and gravimetric water vapour sorption techniques. Int. J. Pharm. 310, 220-229

9. Serajuddin, A.T. (1999) Solid dispersion of poorly water-soluble drugs: early promises, subsequent problems, and recent breakthroughs. J. Pharm. Sci. 88, 1058-1066

10. Pouton, C.W. (2006) Formulation of poorly water-soluble drugs for oral administration: physicochemical and physiological issues and the lipid formulation classification system. Eur. J. Pharm. Sci. 29, 278-287

11. Ghaderi, R. et al. (1999) Preparation of biodegradable microparticles using solutionenhanced dispersion by supercritical fluids (SEDS). Pharm. Res. 16, 676-681

12. Bhugra, C. et al. (2007) Prediction of the onset of crystallization of amorphoussucrose below the calorimetric glass transition temperature from correlations with mobility. J. Pharm. Sci. 96, 1258-1269

13. Weuts, I. et al. (2005) Salt formation in solid dispersions consisting of polyacrylic acid as a carrier and three basic model compounds resulting in very high glass transition temperatures and constant dissolution properties upon storage. Eur. J. Pharm. Sci. 25, 387-393

14. Konno, H. and Taylor, L.S. (2006) Influence of different polymers on the crystallization tendency of molecularly dispersed amorphous felodipine. J. Pharm. Sci. 95, 2692-2705 